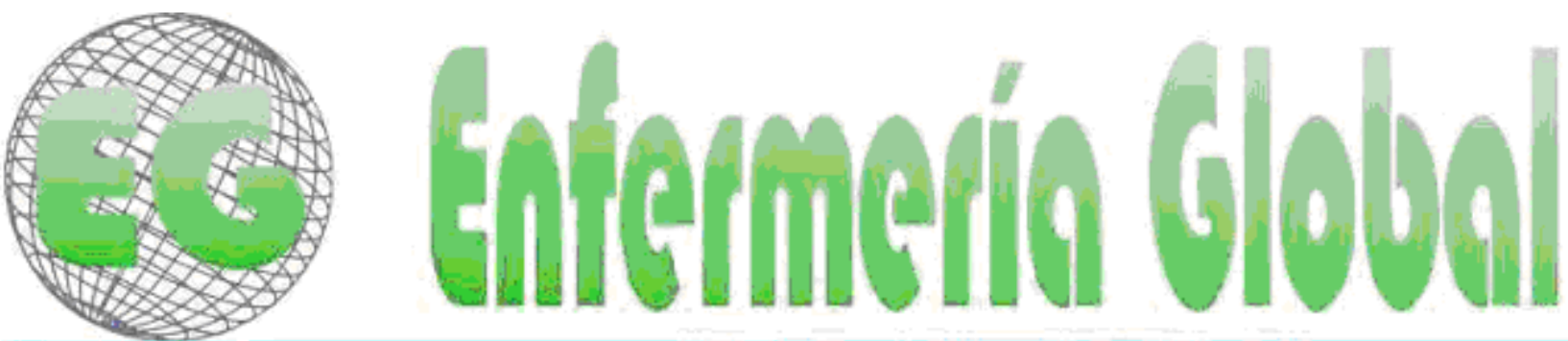

$N^{\circ} 18$

Febrero 2010

www.um.es/eglobal/

ADMINISTRACIÓN - GESTIÓN - CALIDAD

\title{
PROPUESTA DE POLÍTICAS ADMINISTRATIVAS DE RECURSOS HUMANOS PARA EL DEPARTAMENTO DE ENFERMERÍA DE UN HOSPITAL TIPO III DE SAN FELIPE
}

ADMINISTRATION POLICY PROPOSAL OF HUMAN RESOURCES FOR THE NURSING DEPARTMENT OF A TYPE III HOSPITAL IN SAN FELIPE, VENEZUELA.

\author{
*Bazán Orozco, YR., "*Salazar M, M. \\ *Lcdo. en Enfermería. Supervisor General del Hospital Central de San Felipe-Yaracuy, \\ Vicepresidente de la Sociedad Científica en Cuidados Intensivos seccional Yaracuy. ** Phd. \\ en Ciencias de la Educación, Profesora jubilada activa de la FCS de la Universidad de \\ Carabobo. Venezuela.
}

Palabras clave: Políticas, Gerencia, Administración, Recursos Humanos, Enfermería.

Keywords: Policies, Management, Administration, Human Resources, Nursing.

\section{RESUMEN}

La presente investigación, correspondió a un Proyecto Factible apoyado en un estudio de campo a nivel descriptivo, que tuvo como objetivo Diseñar una Propuesta de Modelo de Políticas Administrativas de Recursos Humanos para el Departamento de Enfermería de un Hospital Tipo III de San Felipe. Este estudio fue elaborado en un Hospital público del Estado Yaracuy. La población estuvo constituida por 19 supervisores adscritos al Departamento de Enfermería. Dado que las características de esta población fue pequeña y finita, se tomó como muestra a todos los individuos que la integraron. La recolección de los datos se realizó a través de un instrumento tipo cuestionario estructurado en tres partes; la primera referida a los datos demográficos, con cuatro (4) preguntas de selección simple; la segunda, dirigida a diagnosticar la necesidad de Políticas Administrativas de Recursos Humanos para el Departamento de Enfermería, conformada por veintinueve (29) preguntas cerradas con opciones de respuestas Sí-No, y la tercera parte correspondiente a la medición de factibilidad de mercado, técnico y financiero, conformado por quince preguntas cerradas con opciones de respuesta Sí-NO. Antes de aplicar el instrumento se sometió a una prueba de validez de contenido por juicios de expertos donde se determinó su confiabilidad mediante una prueba piloto, aplicándose a los datos recabados un procedimiento estadístico mediante la prueba de Kuder Richardson 20, que permitió calcular un coeficiente de confiabilidad que arrojó como resultado 0,84 recomendando la aplicación del instrumento a la muestra en estudio. El diagnostico concluyó en la inexistencia de políticas escritas y la necesidad de 
Diseñar una Propuesta de Políticas Administrativas de Recursos Humanos para el Departamento de Enfermería de un Hospital Tipo III. Los estudios de Mercado, Técnico y Financiero, demostraron la factibilidad y viabilidad de la propuesta. Entre las recomendaciones se sugirió la implementación del proyecto.

\section{ABSTRACT}

This research corresponded with a Feasible Project supported in a descriptive field study which had as its objective to design an Administrative Policy Proposal of Human Resources for the Nursing Department of a Type III Hospital in San Felipe. This study was carried out in the public hospital of Yaracuy State. The population was made up of 19 supervisors belonging to the nursing department. Given that this was a small and finite population, all the individuals it includes were taken for the sample. The data collection was made through a questionnaire instrument structured in three parts; the first referring to demographic data with four questions of simple selection; the second directed at diagnosing the necessity of Administrative Policies of Human Resources for the Nursing Department constituted of 29 closed questions with Yes/No answers, and the third part corresponding to the measure of market, technical and financial feasibility constituted of 15 closed questions with answers options Yes/No. Before applying it, the instrument was subjected to a test of validity of its content by experts where its reliability was determined through a pilot test, applying a statistical procedure to the collected data through the Kuder Richardson 20 test, that allowed the calculation of a coefficient of reliability that resulted in 0.84 , recommending the application of the instrument to the sample studied. The diagnosis concluded in the non-existence of written policies and the necessity of designing an Administrative Policy Proposal of Human Resources for the Nursing Department of a Type III Hospital. The market, technical and financial studies showed the feasibility and viability of the proposal. Among the recommendations an implementation of the project was suggested.

\section{INTRODUCCIÓN}

La Administración de Recursos Humanos, no es más que resaltar a las personas como individuos que participan en las organizaciones, poseedoras de un increíble don de crecimiento y desarrollo, no obstante las organizaciones requieren integrar la conducta de sus empleados para evitar el caos y mantener un equilibrio y orden dentro las mismas, así como también la funcionabilidad y estructura, de allí la importancia de lineamientos formalizados los cuales dirijan la acción de pensamiento y resoluciones de problemas comunes relacionados con los objetivos de la organización. Por consiguiente es fundamental que en una Organización de Salud, como un Hospital por ejemplo, existan mecanismos que faciliten el fortalecimiento de estos aspectos tan importantes en su personal. Tales mecanismos no son más que las Políticas de Recursos Humanos.

En América Latina existen grandes diferencias, en cuanto al estado de desarrollo de la práctica y las políticas de Recursos Humanos en enfermería, relacionada y determinada por las condiciones de cada país, ya sean económicas, políticas o sociales. Entre las diferencias resaltan la composición de la fuerza laboral, el estatus social de la profesión, la capacidad organizativa de los diferentes grupos que conforman el personal de enfermería, el nivel educativo de enfermería, tanto de profesionales como auxiliares, y las políticas de salud de las cuales disponen cada país.

Las políticas de salud fueron establecidas en América Latina a mitad del siglo pasado, mediante la extensión de cobertura de los servicios de salud, la cual propulsó la ampliación de las estructuras físicas de los servicios y la incorporación de un grueso número de Recursos Humanos en las diversas ramas del campo de trabajo, incluyendo al personal de enfermería; esto trajo como consecuencia que se incorporara una numerosa cantidad de 
este personal sin preparación específica y adecuada para la práctica requerida, la ausencia de políticas para la preparación de Recursos Humanos de enfermería con metas a corto y mediano plazo, y la inexistencia de una política de administración de personal para fijar salarios y carga horaria adecuada al modelo de atención, tal como lo reveló un documento publicado en Chile por Chompré y col. ${ }^{1}$ denominado Enfermería del Siglo XXI en América Latina, patrocinado por la fundación W.K. Kellogg y con el apoyo del Consejo Internacional de Enfermería.

Chiavenato $^{2}$, refiriéndose a las políticas de Recursos Humanos, señala que "se refieren a la manera como las organizaciones aspiran trabajar con sus miembros para alcanzar por intermedio de ellos los objetivos organizacionales, a la vez que cada uno logra sus objetivos individuales". Entendiéndose con esto, que las políticas constituyen una orientación administrativa fundamental para el trabajador, ya que no solo impiden que realicen funciones que no son de su competencia o para los cuales no están preparadas, sino que se enfoca al logro de las metas y objetivos propuestos.

Ahora bien, el personal de enfermería se desenvuelve dentro del contexto de una organización de salud, y estas tienen políticas orales y/o escritas, sobre el comportamiento o aptitud que han de mantener sus integrantes. Marriner ${ }^{3}$, refiriéndose a las políticas de enfermería señala que "A veces las políticas se generan en los niveles de operación de supervisión y primera línea, y se imponen en forma ascendente". Es decir, que en situaciones excepcionales las políticas aparecen también por solicitud de subalternos, aunque vale señalar, que en ocasiones las políticas podrían ser formuladas simultáneamente por ambas direcciones, es decir, por directivos y trabajadores.

El Departamento de Enfermería constituye unos de los pilares más importantes de un Hospital, es por ello el compromiso del personal en la superación constante, para contribuir de manera significativa a la implementación de nuevas gestiones de salud en servicio que ofrece un Hospital, aportando un excedente a las expectativas del paciente que es quien recibe el Cuidado Humano.

Ahora bien, el investigador en su trabajo diario como supervisor observó en el personal de enfermería de un Hospital Tipo III de San Felipe, indisciplina al momento de ocurrir situaciones administrativas complejas, lo que pudiera estar relacionado con la inexistencia de políticas escritas dentro del Departamento de Enfermería, creándose incertidumbre en el personal e interrumpiendo de alguna manera el cuidado al paciente. Trayendo como consecuencias, por ejemplo, que al ocurrir la ausencia de algún recurso de enfermería de manera imprevista en alguna unidad clínica de cuidado permanente, el personal presente alegue no continuar la guardia, manifestando que no existe nada escrito al respecto que lo comprometa u obligue a continuar ya que nunca lo han observado ni leído en ninguna parte y que además ya cumplieron sus horas reglamentarias, olvidando con esta actitud, que están atendiendo seres humanos hospitalizados, que asisten al Hospital en busca de ayuda para su enfermedad y que dependen algunas veces de un muy elevado porcentaje del cuidado de enfermería, tales como pacientes en ventilación mecánica, intervenciones quirúrgicas de urgencia, partos, ingresos a las Áreas de Emergencia de Adultos o Pediátrica, entre otras.

De la misma manera, se evidenció un éxodo voluntario de Recursos Humanos de enfermeras(os) contratadas(os) de reciente ingreso a la institución y por consiguiente con poco tiempo en su desempeño laboral. Esta situación llamó la atención por su frecuencia y porque ocurren más en áreas específicas como Emergencia Adultos y Sala de Partos, generando turnos solos que repercuten en sobrecarga laboral y guardias extras en el 
personal asistencial. Todo ello, debido probablemente a que el personal de enfermería de nuevo ingreso, no se adapta al campo de trabajo donde está laborando, no se siente lo suficientemente comprometido con el Departamento de Enfermería o simplemente la institución no ha podido satisfacer las expectativas traídas por el trabajador, debido a que no existe una política de socialización para el cargo. No obstante se comprende, que todas las áreas clínicas, deben tener políticas de enfermería escritas; sin embargo, existen algunas áreas o unidades clínicas, donde se hace más notable la necesidad de Formulación de Políticas de Recursos Humanos. Referente a esto, Huber ${ }^{4}$ manifiesto que "son áreas donde existe confusión acerca del origen de la responsabilidad o donde la ausencia de orientación pudiera resultar en negligencia, mal desempeño o practica errónea de una acción necesaria para el bienestar del paciente".

Otro aspecto a considerar, es la ausencia de políticas escritas en la Selección de Recursos Humanos para el Departamento de Enfermería, tomando en cuenta la adaptación al cargo y su desempeño laboral, tal como explica Chiavenato ${ }^{2}$ al señalar que "la tarea básica de selección es escoger entre los candidatos reclutados aquellos que tengan mayores probabilidades de adaptarse al cargo ofrecido y desempeñarlo bien". Ambas teorías descritas por estos autores, no hacen más que reforzar lo importante que es contar con Políticas de Recursos Humanos escritas que puedan tomarse como lineamientos en las distintas áreas del Departamento de Enfermería, ya que es fundamental que todas las personas se adhieran a un mismo patrón de decisión dadas ciertas circunstancias.

Por otra parte, el investigador, como observador participante activo en el contexto laboral de enfermería, observó el desinterés y el déficit de conocimiento que manifiesta el personal de enfermería en su quehacer diario en cuanto a las políticas de enfermería; evidentemente no se realizan programas educativos periódicamente, talleres o cursos de actualización dentro de la institución referente a las necesidades de las políticas de enfermería, ocasionando en cierto modo apatía en el personal al sentirse aislado a pesar de ser conscientes de que persiguen un mismo fin, como es brindar cuidado humano integral a los pacientes que asisten a las diferentes unidades clínicas de atención. Esta actitud desinteresada del personal de enfermería y la ausencia de Políticas Administrativas para el Departamento de Enfermería, afecta el desarrollo de los objetivos trazados por la Organización de Salud, el Departamento de Enfermería y del personal, de modo que surge la necesidad de establecer lineamientos administrativos que sirvan de guía para la acción y para dar respuesta a situaciones que puedan presentarse.

Así mismo, ocurren discrepancias en las relaciones laborales de enfermería, como enfrentamientos al tomar algunas decisiones administrativas, ya que no poseen lineamientos específicos que los guíen claramente, así vemos por ejemplo, que a la hora de levantar un acta de retardo algunos supervisores de enfermería lo hacen diez minutos luego de la hora de entrada y otros hasta veinte minutos después de esa hora, dando lugar a reclamos del personal subalterno al supervisor, en este último caso, ya que alegan que el día anterior otra supervisora o supervisor no sancionó a alguna colega que llegó a la misma hora. Evidenciándose que no existe uniformidad de criterios en las políticas verbales existentes en el Hospital. Esta contradicción de criterios crea incertidumbre y contribuye a sentimientos de parcialidad, trato preferencial e injusticia en el personal de enfermería. En este sentido, Marriner $^{3}$ señaló que "se eleva la moral cuando el personal comprende que está siendo tratado con ecuanimidad". De tal modo que vale la pena aclarar, que la justicia es una característica fundamental atribuida a la aplicación de las políticas.

Entre tanto, los hechos como la rotación de puestos al personal de enfermería del área donde labora, sin avisar o insuficiente tiempo de notificación y sin tomar en consideración su 
experiencia y vocación de servicio en el área, también dan cuenta de la falta de uniformidad de criterios e inexistencia de políticas administrativas escritas y solo logra que el personal lo vea como un castigo y no como parte de su desarrollo profesional y laboral para el logro de una capacitación integral. Otro ejemplo que refuerza lo antes señalado por el autor, es referente a que se observó en algunas áreas clínicas, e incluso se lee en algunos reportes del libro de supervisión, referencias acerca de la presentación del personal de enfermería quienes han asumido el uso de monos estampados y/o extremadamente cortos o ceñidos al cuerpo, dejando de lado el uniforme clásico que ha identificado a la enfermera o enfermero, sumado a ello el empleo de accesorios muy extravagantes como collares, pulseras y zarcillos, inadecuados para el tipo y naturaleza del trabajo desempeñado.

Todo lo expresado deja ver la inexistencia de políticas que regulen no solo la práctica de enfermería, sino también las formas de presentación, además de la necesidad de lineamientos administrativos escritos en el Departamento de Enfermería, donde pueda guiarse y canalizarse un pensamiento de acción para la toma de decisiones, considerándose pertinente como Objetivo principal: Diseñar una Propuesta de Políticas Administrativas de Recursos Humanos para el Departamento de Enfermería de un Hospital Tipo III de San Felipe, y como Objetivos específicos: Diagnosticar las características de las Políticas Administrativas de Recursos Humanos para el Departamento de Enfermería de un Hospital Tipo III de San Felipe, en función a los constructos teóricos sobre el clima organizacional y realidad de la práctica de enfermería, Determinar la Factibilidad de Mercado, Técnico y Financiero de Políticas Administrativas de Recursos Humanos para el Departamento de Enfermería de un Hospital Tipo III de San Felipe y proponer el Diseño de un Modelo de Políticas Administrativas de Recursos Humanos para el Departamento de Enfermería de un Hospital Tipo III de San Felipe.

\section{MATERIAL Y MÉTODO}

El presente trabajo de investigación se ubicó en la modalidad proyecto factible, la cual de acuerdo al Manual de Trabajos de grado de Especialización y Maestría y Tesis Doctorales de la Universidad Pedagógica Experimental (UPEL) ${ }^{5}$ "consiste en la investigación, elaboración y desarrollo de una propuesta de un modelo operativo viable para solucionar problemas, requerimientos o necesidades de organizaciones o grupos sociales; puede referirse a la formulación de políticas, programas, tecnologías, métodos o procesos". Así mismo es importante decir que un proyecto factible debe tener apoyo en una investigación de campo, documental o un diseño que incluya las dos modalidades anteriores.

En tal sentido, se desarrollaron en este trabajo aspectos relativos al tipo de estudio y diseño de investigación, anexándolos en base a los objetivos planteados, como este proyecto factible; apoyándose en un estudio de campo, fundamentado en una investigación documental y bibliográfica, las cuales sustentan teóricamente la propuesta. El estudio se desarrolla en tres fases: Fase I. Diagnostica, Fase II. Factibilidad y Fase III. Diseño de la Propuesta.

\section{Tipo de Investigación}

De acuerdo al problema planteado, referido a la Propuesta de Políticas Administrativas de Recursos Humanos para el Departamento de Enfermería de un Hospital tipo III, y en función a sus objetivos, se incorporó el tipo de investigación denominado Proyecto Factible, el cual consiste en una propuesta orientada a resolver un problema en los Recursos Humanos de enfermería y a la vez mejorar la calidad del cuidado al paciente como ente final de las actividades del recurso de enfermería. 
De igual manera la investigación correspondió a un diseño descriptivo, el cual según Hurtado ${ }^{6}$, "tiene como objetivo la descripción precisa del evento de estudio, con el propósito de enumerar detalladamente las características del fenómeno". En atención a esta modalidad de investigación se introdujeron tres grandes fases en el estudio, a manera de cumplir con los requisitos involucrados en un proyecto factible. En la primera de ellas, inicialmente se desarrolló un diagnóstico de la situación existente sobre la necesidad de Diseñar las Políticas Administrativas de Recursos Humanos para el Departamento de Enfermería, en la segunda fase del proyecto, y atendiendo a los resultados del Diagnóstico, se formuló la Factibilidad de la propuesta, relacionada a los Estudios de Mercado, Técnico y Financiero, la tercera fase se refirió al Diseño de Políticas Administrativas Recursos Humanos para el Departamento de Enfermería de un Hospital tipo III de San Felipe.

\section{Diseño de la Investigación}

Atendiendo los objetivos de manera primaria, la investigación se orientó hacia la incorporación de un diseño de campo no experimental, por cuanto este diseño de investigación permite no solo observar, sino recolectar los datos directamente de la realidad objeto de estudio, para posteriormente analizar e interpretar los resultados de estas indagaciones. Se considera de campo atendiendo a la definición de Sierra, citado por Ramírez ${ }^{7}$, que la señala como "el tipo de investigación a través del cual, se estudian los fenómenos sociales en su ambiente natural" y según la Universidad Nacional Abierta $(\mathrm{UNA})^{8}$, que la define como un "proceso sistemático, riguroso y racional de recolección, tratamiento, análisis y presentación de datos basado en la estrategia de recolección directa de la realidad, de las informaciones necesarias para la investigación". El diseño es una estrategia que orienta y facilita las etapas de la investigación, que en el caso de los proyectos factibles hace referencia a las tres fases señaladas por Cerda, $\mathrm{H}^{9}$, referidas al Diagnóstico, Factibilidad y Diseño de la Propuesta.

\section{Fases del Proyecto Factible}

A fin de operacionalizar el presente estudio por medio de esta modalidad, cabe destacar que solo se desarrollaron sus tres primeras fases, es decir la fase I, referente al Estudio Diagnostico, la fase II, referente al Estudio de Factibilidad y la fase III, referente al Diseño de la Propuesta. Las otras dos fases, de Ejecución y Evaluación corresponden a las autoridades competentes, tal como lo señalan Orozco, Labrador y Palencia (2002) ${ }^{10}$ cuando afirman que "El desarrollo del Proyecto Factible en trabajos de Grado y Maestría debe contemplar tres fases: Diagnóstico, Factibilidad y Desarrollo de la propuesta. En la tesis doctoral además de estas fases necesariamente debe incluir las etapas de Ejecución y Evaluación". A continuación se describen las tres primeras fases.

\section{Fase I: Estudio Diagnóstico}

Según Ander-Egg"11, el Diagnostico es "el análisis y determinación de una situación sobre la base de informaciones y hechos recogidos y ordenados sistemáticamente", de allí que esta fase del diseño se efectúa mediante la aplicación de un instrumento de recolección de datos tipo cuestionario, el cual permite medir la variable investigada. A través de esta fase se determina la puesta en marcha del proyecto, es decir se realiza un diagnóstico para determinar la necesidad de Provisión, Aplicación, Mantenimiento, Desarrollo y Control de Recursos Humanos, como políticas escritas para el Departamento de Enfermería. Esta 
consulta se ejecuta por medio de una investigación de campo a todas las personas que serían beneficiadas con la ejecución del proyecto.

\section{Fase II: Estudio de Factibilidad}

A través de esta fase, se determina de manera científica la viabilidad del proyecto; asimismo se miden, se cuantifican y se relacionan los resultados parciales de los Estudios de Mercado, Técnico y Financiero. Para Gómez, C. ${ }^{12}$, la Factibilidad "representa la posibilidad de llevar a cabo un proyecto, tomando en cuenta la necesidad identificada, los costos y beneficios del proyecto, los recursos técnicos y humanos, financiamiento, estudios de mercado, beneficiarios del proyecto". Según la precitada autora deben tenerse en cuenta tres objetivos fundamentales en toda elaboración de proyectos como son: verificar que existe un mercado potencial insatisfecho y que es viable, demostrar que tecnológicamente es posible producirlo y que se cuenta con todos los insumos necesarios, y por último demostrar que es económicamente rentable llevar a cabo su realización.

El Estudio de Mercado, se refiere esencialmente a la determinación y cuantificación de la demanda y la oferta. Su finalidad en sí es probar que existe un número suficiente de personas, instituciones y empresas u otras entidades que dadas ciertas condiciones demuestren una demanda que justifica la puesta en marcha del proyecto. La cuantificación de la oferta y la demanda se obtendrá por medio de encuestas.

En cuanto al Estudio Técnico, debe demostrar la viabilidad técnica del proyecto, además de demostrar y justificar cual es la alternativa que mejor se ajusta a los criterios de optimización que corresponda aplicar al proyecto.

Por su parte, el Estudio Financiero determina la manera de obtener los recursos económicos para cubrir los costos del proyecto; esto se desarrolla partiendo del análisis de costo, estimando el capital disponible, y luego estableciendo un plan de financiamiento para cubrir las necesidades totales de capital para determinar la vialidad financiera del proyecto.

\section{Fase III: Diseño de la Propuesta}

Esta fase se elaboró en base a los documentos del Diagnóstico, para lo cual se tomaron en cuenta las necesidades del universo investigado. De igual manera el diseño de las políticas incluye: Presentación, Justificación, Fundamento Teórico, Fundamento Legal, Objetivos, Propuesta en sí de las políticas de enfermería, Recursos Necesarios, Seguimiento, Evaluación y Anexos.

\section{Población}

De acuerdo a Selltiz, Jahoda y Cook ${ }^{13}$, "Una población es un conjunto de todos los casos que concuerdan con una serie determinada de especificaciones", en el caso que nos ocupa, la población estuvo conformado por 19 enfermeras adscritas al Departamento de Enfermería de un Hospital tipo III de San Felipe.

Este universo constituyó una población de tipo finita, la cual Ramírez ${ }^{7}$ definió como "Aquella cuyos elementos en su totalidad son identificables por el investigador. Por lo menos desde el punto de vista del conocimiento que se tiene sobre su cantidad total". 


\section{Muestra}

En el presente estudio se tomó al grupo de diecinueve (19) supervisoras de enfermería, como una muestra censal, que según Seijas ${ }^{14}$, "supone la obtención de datos de todas las unidades del universo acerca de las cuestiones que constituyen el objeto del censo; los datos se recogen entre muestras de unidades que representan el universo dado, porque la población es pequeña". Es decir que dada las características de esta población pequeña y finita, se tomaron como unidades de estudio e indagación a todos los individuos que la integraron.

\section{Técnica e Instrumento de Recolección de Datos}

En este trabajo se empleó la encuesta, definida por Polit y Hungler ${ }^{15}$ como "cualquier actividad de investigación en la que se recogen datos de una porción de la población con el fin de examinar sus características, opiniones o intenciones".

De igual manera para la aplicación del instrumento de recolección de datos se utilizó el procedimiento entrevistas formalizadas, que de acuerdo a Sabino ${ }^{16}$, "se desarrollan con base en un listado fijo de preguntas, cuyo orden y redacción permanecen invariable y que comúnmente se administra a un gran número de entrevistados para su posterior tratamiento estadístico".

Referente al instrumento para recabar la información, se elaboró un instrumento tipo cuestionario estructurado en dos partes, la primera parte está conformada por veintinueve (29) preguntas cerradas con dos opciones de respuesta y/o dicotómicas, Sí o NO, dirigidas a diagnosticar la necesidad de políticas de Recursos Humanos para el Departamento de Enfermería. La segunda parte, conformada por quince (15) preguntas cerradas con dos opciones de respuesta Sí-NO que permitió medir la Factibilidad de Mercado, Técnico y Financiero. Antes de aplicar el instrumento fue pertinente la validez y confiabilidad del mismo.

\section{Validez}

Según Pólit y Hungler ${ }^{15}$, la validez es "el grado en que un instrumento mide lo que se supone debe medirse", por lo tanto teniendo presente dicho concepto se revisó la variable según otras investigaciones, y se consultó a estudiosos familiarizados con la temática, razón por la cual se utilizó el juicio de expertos.

\section{Confiabilidad}

En esta investigación se aplicó una prueba piloto, la cual según Pick y López ${ }^{17}$, "es un estudio previo al estudio final; es una especie de ensayo de dicho estudio. Para llevarlo a cabo, el investigador no tiene un conocimiento preciso de qué variables se van a controlar". Esta prueba piloto se ejecutó con una muestra inferior a la definitiva, en este caso a ocho (08) sujetos que comparten las mismas características del universo estudiado y que laboran en un Hospital público del Estado Lara, en el Departamento de Enfermería como supervisoras de enfermeras.

Los datos recabados con el pilotaje fueron tabulados y sometidos a procedimientos estadísticos mediante la prueba de Kuder Richardson 20 (KR20) a las interrogantes dicotómicas propuestas por el autor en referencia, que permitió calcular el coeficiente de 
confiabilidad. El mismo arrojó un resultado de 0,84 catalogado como bueno y recomendando la aplicación del instrumento a los constituyentes de la muestra del estudio a realizar.

\section{Técnica de Análisis de Datos}

Una vez recolectada la información a través de la aplicación del instrumento de recolección de datos y obteniendo un cúmulo de información fundamental, se procedió a organizarla, tabularla y codificarla, a manera de describir o explicar las posibles tendencias que se puedan reflejar. Para Pólit y Hungler ${ }^{15}$, el análisis de datos es la "organización sistemática y síntesis de los datos de la investigación, así como prueba de la hipótesis de la investigación mediante dichos datos", de allí que en la presente investigación se realizaron cálculos estadísticos como la tabulación, frecuencia de respuestas, porcentajes y media porcentual, con el propósito de hacer un análisis o síntesis cuantitativo sustentados en base al constructos teórico de la investigación.

\section{RESULTADOS Y DISCUSIÓN}

\section{Resultados del Estudio Diagnóstico}

Por medio de este Estudio Diagnostico se determinó la necesidad de la formulación de un Diseño de Políticas Administrativas de Recursos Humanos para el Departamento de Enfermería de un Hospital tipo III, en cuanto a la Provisión, Aplicación, Mantenimiento, Desarrollo y Control de Recursos Humanos de Enfermería, a través de un instrumento de recolección de datos tipo cuestionario dicotómico aplicado a los diecinueve supervisores adscritos al Departamento de Enfermería. Para ello se consideraron dos aspectos fundamentales.

El Primer aspecto estuvo relacionado con el Objetivo del Proyecto, referente al Diseño de Políticas administrativas de Recursos Humanos para el Departamento de Enfermería; el Propósito del proyecto, relativo a proveer a los supervisores de lineamientos escritos que guíen la toma de decisiones y acciones a seguir, y a la Caracterización del Proyecto, que comprende la Naturaleza del Proyecto, la Importancia del Proyecto y la Ubicación Sectorial del proyecto.

Referente al segundo aspecto, este correspondió a la Presentación, Análisis e Interpretación de los Datos del instrumento aplicado a la muestra, en donde al interrogarse sobre la existencia de políticas administrativas de Recursos Humanos de Enfermería, los resultados se reflejaron negativamente en los siguientes Factores; el ochenta y tres con cincuenta y cinco por ciento (83.55\%) en cuanto a la Provisión de Recursos Humanos de Enfermería, el sesenta y cinco con setenta y ocho por ciento (65.78\%) en cuanto a la Aplicación de Recursos Humanos de Enfermería, el setenta y nueve con ochenta y dos por ciento $(79,82 \%)$ en cuanto al Mantenimiento de Recursos Humanos de Enfermería, cerca del cien por ciento (94.73\%) en cuanto al Desarrollo de Recursos Humanos de Enfermería, y un ochenta y seis con dieciocho por ciento (86.18\%) en cuanto al Control de Recursos Humanos de Enfermería.

Por consiguiente, tomando en consideración el valor porcentual de cada uno de los factores de la Variable Políticas Administrativas de Recursos Humanos Para el Departamento de Enfermería de un Hospital Tipo III, se corroboró que el ochenta y dos por ciento (82\%) de la población estudiada confirmó la inexistencia de políticas de Enfermería en este sentido, ratificándose con estos resultados la necesidad de diseñar una propuesta de Políticas Administrativas de Recursos Humanos para el Departamento de Enfermería de un 
Hospital Tipo III, que brinde una alternativa de solución viable a un problema institucional real.

\section{Resultados del Estudio de Mercado}

De acuerdo a los datos obtenidos en el Estudio Diagnóstico, el ochenta y dos por ciento $(82 \%)$ de la población estudiada confirmó la inexistencia de políticas administrativas de Recursos Humanos para el Departamento de Enfermería, manifestándose de esta manera una alta demanda sobre la necesidad de diseñar un conjunto de lineamientos los cuales servirían de herramienta administrativa como alternativa de solución viable a una necesidad presente en el Departamento de Enfermería. Así mismo, en cuanto a la factibilidad de llevar a cabo este proyecto, las encuestas aplicadas a los diecinueve supervisores adscritos al Departamento de Enfermería, confirmaron cerca del noventa por ciento (89\%) una alta posibilidad real de llevar a cabo esta propuesta de diseño y de establecerse en el mercado, quedando así demostrado que la oferta satisface a la demanda por cuánto existen recursos humanos beneficiados, tales como; supervisores de enfermería, personal de enfermería a cargo de los supervisores y los pacientes recluidos en el centro hospitalario, lo que en consecuencia significa que es viable desde el punto de vista de mercado el diseño de la propuesta.

\section{Resultados del Estudio Técnico}

Para la realización del estudio técnico, se tomaron en consideración cinco elementos importantes del proyecto, tales como el tamaño, las fortalezas, la localización física, el proceso de transformación y el análisis de costos, demostrándose por medio de cada uno de ellos que técnicamente es viable el desarrollo del Diseño de Políticas Administrativas de Recursos Humanos para el Departamento de Enfermería de un Hospital tipo III de San Felipe, y que además se cuenta con los insumos necesarios para su realización, ya sea de Recursos Humanos con la participación de expertos en materia de políticas de salud, o Recursos Materiales al disponer de un auditórium acondicionado con mesas, sillas, pizarrón, pantalla de proyección, video been, retroproyector y computadoras, entre otros.

De esta manera, al determinar la inversión, representada por los recursos humanos, materiales y de servicios, se observó que muchos de éstos elementos se encuentran disponibles dentro de la institución de salud y que pueden ser tramitadas a través del Departamento de Enfermería a la dirección del Hospital Central de San Felipe. De tal modo que se puede inferir, que es posible la elaboración del proyecto del diseño propuesto, al demostrarse que se cumple con los pasos requeridos desde el punto de vista técnico del estudio realizado.

\section{Conclusiones del Estudio Financiero}

Por medio del estudio financiero, se determinó la forma de obtener los recursos económicos necesarios para cubrir los costos del proyecto de Diseño de Políticas Administrativas de Recursos Humanos para el Departamento de Enfermería de un Hospital tipo III. De esta manera, partiendo del análisis de costos se precisaron las Necesidades Totales de Capital, las cuales fueron de 1.750.600 Bs. (1750,6 Bs. F), y la Estimación del Capital Disponible, donde se consideró los activos tangibles, equivalente a 1.254.400 Bs. (1.254,4 Bs. F) representados por los recursos humanos, materiales y de servicios de la institución, y los activos intangibles equivalente a 470.000 Bs. (470 Bs. F) del aporte de los participantes en el proyecto del diseño, de allí que el capital disponible para la elaboración del proyecto sea de 
1.729.000 Bs. (1.729 Bs. F). Es por ello, se que se desarrolló un valioso Plan de Financiamiento, con el propósito de cubrir las necesidades totales de capital y quedar demostrado así la viabilidad financiera del proyecto.

\section{PROPUESTA DE DISEÑO DE LA PROPUESTA}

En esta fase del proyecto factible, se presenta el Diseño de un Modelo de Políticas Administrativas de Recursos Humanos para el Departamento de Enfermería de un Hospital Tipo III de San Felipe de una manera estructural y enfocado desde un punto de vista sistémico, específicamente como un sistema abierto, ya que los elementos que la componen reflejan entre sí relaciones que pueden ser objetivadas, y el conjunto de esos elementos interrelacionados constituyen un todo estructurado, el cual se propone ser estudiado desde su conceptualización como Políticas Administrativas de Recursos Humanos de Enfermería según las transformaciones ocurridas en su Planificación, Ejecución, Evaluación y Control, considerándose ésta como un proceso en continua transformación (retroalimentación).

\section{Objetivo General}

Proporcionar a los supervisores del Departamento de Enfermería de un Hospital Tipo III de San Felipe, el Diseño de un Modelo de Políticas Administrativas de Recursos Humanos para su desarrollo e implementación, que garantice el éxito de la gestión administrativa y el alcance de los objetivos propuestos.

\section{Objetivos Específicos}

1.-Tramitar ante la institución de salud y el Departamento de Enfermería, las autorizaciones y/o permisos necesarios, que faciliten el desarrollo e implementación del Diseño de un Modelo de Políticas Administrativas de Recursos Humanos para el Departamento de Enfermería de un Hospital Tipo III de San Felipe.

2.-Solicitar ante la institución de salud y el Departamento de Enfermería, los Recursos Humanos, Materiales y de Servicios que disponga la institución, a manera de que se facilite el desarrollo e implementación del Diseño de un Modelo de Políticas Administrativas de Recursos Humanos para el Departamento de Enfermería de un Hospital Tipo III de San Felipe.

3.-Desarrollar un Plan de Motivación dirigido a los supervisores de enfermería, a manera de sensibilizarlos y concienciarlos acerca de la necesidad del desarrollo e implementación del Diseño de un Modelo de Políticas Administrativas de Recursos Humanos para el Departamento de Enfermería de un Hospital Tipo III de San Felipe.

4.-Elaborar un Programa de financiamiento, que garantice el capital necesario para el desarrollo e implementación del proyecto.

5.-Determinar las Políticas Administrativas de Recursos Humanos para el Departamento de Enfermería de un Hospital tipo III de San Felipe, de acuerdo al contexto social, diagnóstico realizado y conocimientos teóricos adquiridos por los participantes, para la formulación.

6.-Concretar el Diseño de un Modelo de Políticas Administrativas de Recursos Humanos para el Departamento de Enfermería de un Hospital tipo III de San Felipe, para su presentación ante las autoridades correspondientes. 


\section{Descripción del Diseño de la Propuesta}

La estructura del modelo de Políticas Administrativas de Recursos Humanos para el Departamento de Enfermería de un Hospital Tipo III se fundamenta íntegramente en el Modelo del Sistema Integral de Enseñanza y Aprendizaje de Alberto Block ${ }^{18}$, por lo tanto parte de dos puntos específicos como son los Objetivos y Políticas Institucional de Salud y el Análisis de la Realidad; ambos elementos permiten el establecimiento de Alternativas de Solución frente a las necesidades y a una Estrategia General para satisfacer dichas necesidad información bajo un enfoque sistémico vinculado a su contexto social.

En lo esencial, los Objetivos y Políticas Institucional de Salud se refieren en primer lugar a las metas que persiguen la Institución de Salud y el Departamento de Enfermería, y en segundo lugar a las políticas que norman su actuación, representadas a través del análisis de la Constitución de la República Bolivariana de Venezuela de $1999^{19}$, en su Capitulo V, de los Derechos Sociales y de las Familias, Artículo 84. Del análisis de la Ley del Estatuto de la Función Pública del $2002^{20}$, relacionados a políticas de salud. Del análisis de la Ley del Ejercicio Profesional de la Enfermería del $2005^{21}$, en el Capítulo IV de los Deberes y Derechos de los o las Profesionales de la Enfermería, Articulo 14, parágrafo cinco. Del análisis de la III Convención Colectiva de Trabajo entre el Ministerio de Salud y los Institutos Autónomos a él Adscritos y la Federación de Colegios de Enfermeras(os) de Venezuela del 2002, y del análisis del Código Deontológico de Enfermeras (os) de Venezuela de 1999, en el Capítulo III, relacionado a los Deberes profesionales, Articulo 12, parágrafo 13.

En cuanto al Análisis de la Realidad, en la misma se determina primeramente la necesidad social e institucional, que para la investigación aquí desarrollada viene dada por la ausencia de políticas administrativas de recursos humanos para el Departamento de Enfermería de un Hospital tipo III y la necesidad de diseñarlas. En segundo lugar, a los requerimientos de recursos humanos, materiales y financieros, y en tercer lugar a la investigación actual, donde se consideran la naturaleza de la investigación, el tipo de investigación, la metodología de la investigación y las áreas, líneas y alcance de la investigación.

Referente a las Alternativas de Solución, éstas se establecen luego de la confrontación del análisis de las necesidades, el análisis de los recursos y el análisis de la investigación actual. De allí se prevé una estrategia general con su cuantificación presupuestaria, análisis de la inversión y evaluación de factibilidad sobre la cual debe trazarse el Modelo de Políticas Administrativas de Recursos Humanos para el Departamento de Enfermería.

En cuanto a la Adopción de un Plan, el mismo viene dado, en principio, por el estudio de la realidad, expresado para este caso en ausencia de políticas administrativas de recursos humanos para el Departamento de Enfermería, y en segundo lugar a las alternativas de solución, referidas al diseño de un modelo de políticas. Dentro del plan destacan aspectos; administrativos, de motivación, financiamiento, determinación de las políticas y de diseño de las políticas. El plan debe contener una amplia descripción de todos estos aspectos señalados para la formulación de las políticas deseadas.

Con respecto al Plan de Actividades, aquí es donde se describen todas y cada unas de las operaciones que se deben poner en práctica para lograr los objetivos trazados, enmarcados dentro de los recursos humanos, materiales y financieros. Dentro de este plan de actividades relacionadas al Diseño de Políticas Administrativas de Recursos Humanos para el Departamento de Enfermería destacan las solicitudes de autorización tanto a la Institución de Salud como al Departamento de Enfermería, así como de Recursos humanos, materiales y de servicios. Las actividades de sensibilización, adiestramiento y capacitación a los 
supervisores. Las actividades para el financiamiento interno y externo. Las actividades relacionadas a la formulación de las políticas, y finalmente las actividades donde se concretará la propuesta del diseño de políticas de Enfermería para su presentación a las autoridades competentes.

Finalmente, se realiza la Evaluación, la cual se hace durante todo el proceso, estando presente en las etapas de Diagnóstico, planeación, ejecución, evaluación y control de los resultados, a manera de monitorear el cumplimiento de los objetivos trazados. De la evaluación se podrían hacer reajustes en caso de ser necesario. La evaluación global del sistema Facilita el proceso de retroalimentación del Diseño de Políticas Administrativas de Recursos Humanos para el Departamento de Enfermería con su contexto social.

DIGEAO DE UN MODEO DE POUTCAB AOWNUTTRATVA BDE RECURDOS HUMANOS PARA EL DEPARTAMENTO DE EVIERUERLA DE UNHOUPTAL TROO FUENTE: ALBERTO BLOCK, LADLLERTIO CHAVENATO. ADAPTACION: YOVANY BAZZAN

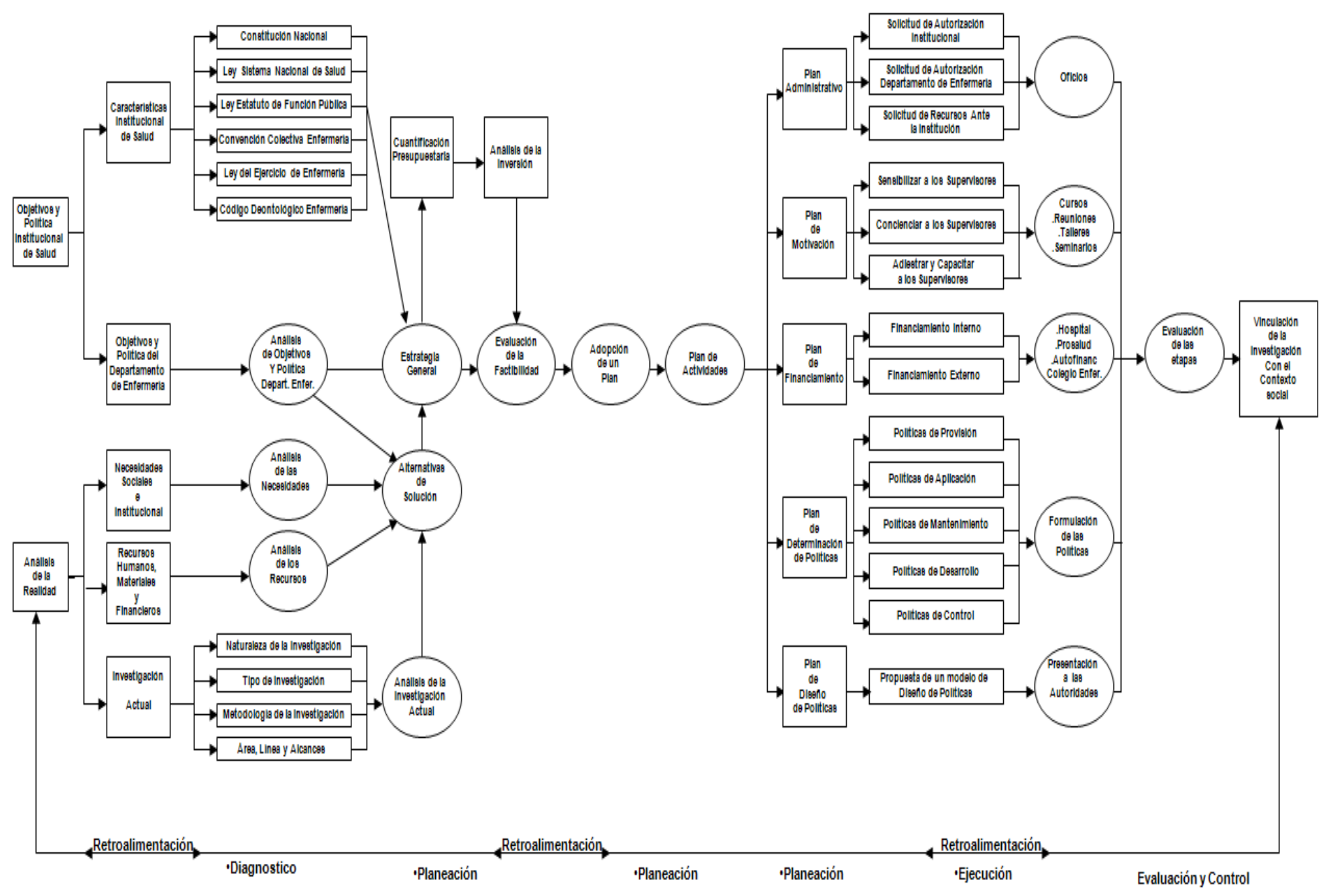




\section{CONCLUSIONES}

A partir del proceso de investigación realizado para la elaboración de la propuesta, enmarcado en los constructos teóricos de la Administración de Recursos Humanos y la realidad de la práctica diaria de enfermería en cuanto a sus políticas, se dio respuestas a los objetivos planteados en este Proyecto Factible, lo que conllevó a obtener como producto final una propuesta de Diseño de Políticas Administrativas de Recursos Humanos para el Departamento de Enfermería de un Hospital Tipo III, representada en la adaptación estructural del Modelo de Sistema Integral de Enseñanza Aprendizaje (SIEA) de Alberto Block. De allí, que analizados los datos obtenidos del Estudio Diagnóstico y a la luz de los Objetivos y teorías que sustentaron la propuesta, se deducen las siguientes conclusiones:

1.1.-Al diagnosticar las características de las Políticas Administrativas de Recursos Humanos para el Departamento de Enfermería de un Hospital Tipo III, tomando en consideración el valor porcentual de cada uno de los factores de la variable, se determinó que el ochenta y dos por ciento $(82 \%)$ de la población encuestada confirmó la inexistencia de políticas escritas en cuanto a Provisión, Aplicación, Mantenimiento, Desarrollo y Control de Recursos Humanos de Enfermería, ratificándose con estos resultados, la necesidad de Diseñar una propuesta de Políticas Administrativas de Recursos Humanos para el Departamento de Enfermería de un Hospital Tipo III, como alternativa de solución viable a la problemática existente.

1.2.-Al determinar la Factibilidad de llevar a cabo el Diseño de Políticas Administrativas de Recursos Humanos para el Departamento de Enfermería de un Hospital Tipo III, la población encuestada confirmó en un ochenta y ocho con setenta y seis por ciento $(88,76 \%)$, la viabilidad de llevar a cabo la propuesta, tomando en consideración la necesidad identificada, los costos y beneficios del proyecto, los recursos técnicos y humanos, financiamiento, estudio de mercado y beneficiarios del proyecto.

1.3.-Las encuestas aplicadas a los diecinueve supervisores adscritos al Departamento de Enfermería, confirmaron cerca del noventa por ciento (89\%), una alta posibilidad real de llevar a cabo esta propuesta de diseño y de establecerse en el mercado, quedando así demostrado que la oferta satisface a la demanda, por cuanto existen Recursos Humanos favorecidos, tales como supervisores de enfermería, personal de enfermería a cargo de los supervisores y pacientes recluidos en el centro hospitalario, lo que en consecuencia significa que es viable desde el punto de vista de mercado el diseño de la propuesta.

1.4.-Al determinar la inversión, representada por los Recursos Humanos, materiales y de servicios, se comprobó que muchos de estos elementos se encuentran disponibles dentro de la institución de salud y que pueden ser tramitadas a través del Departamento de Enfermería a la dirección del Hospital. De tal modo que es técnicamente viable la elaboración del diseño propuesto al demostrarse que se cuenta con los insumos necesarios para su realización, tales como personal experto en materia de políticas de salud y auditórium acondicionado con mesas, sillas, pizarrón, pantalla de proyección, video beem, retroproyector y computadoras.

1.5.-El Estudio Financiero realizado, determinó el presupuesto necesario para el desarrollo del Diseño de Políticas Administrativas de Recursos Humanos para el Departamento de Enfermería y la manera de cómo obtener esos recursos económicos a través de un plan de financiamiento que cubre las necesidades totales de capital, quedando demostrado de esta manera la viabilidad financiera del proyecto. 
1.6.-A partir del basamento teórico de la Administración de Recursos Humanos propuesta por Chiavenato, la adaptación estructural al Modelo de Sistema Integral de Enseñanza Aprendizaje de Block y la realidad de la práctica diaria de enfermería, se elaboró un Diseñó de Políticas Administrativas de Recursos Humanos para el Departamento de Enfermería de un Hospital tipo III, para su presentación ante las autoridades correspondientes, a manera de ofrecer una alternativa de solución viable a un problema real presentado.

\section{BIBLIOGRAFÍA}

1. Chompre R, De Montenegro E, Lange I, Enfermería del Siglo XXI en América Latina. Chile. Fundación W.K. Kellogg. Technicolor Ltda, 1991.

2. Chiavenato I. Administración de Recursos Humanos. Tercera Edición. Bogotá. Editorial McGraw-Hill, 1999. p.162-238.

3. Marriner A. Manual Para la Administración de Enfermería. México. Segunda Edición en Español. Editorial Interamericana, 1984. p.20-21.

4. Huber D. Liderazgo y Administración en Enfermería. México. Editorial McGraw-Hill Interamericana, 2000. p.208.

5. Universidad Pedagógica Experimental Libertador. Manual de Trabajos de Grado de Especialización y Maestría y Tesis Doctorales. Caracas. Tercera Edición. Editado por la UPEL, 2003. p. 16.

6. Hurtado J. El Proyecto de Investigación. Un Enfoque Holístico. Caracas. SYPAL, 2000. p. 77.

7. Ramírez T. Como Hacer un Proyecto de Investigación. Caracas. Editorial Panapo, 1999. p. $76-92$.

8. Universidad Nacional Abierta. Técnicas de Documentación e Investigación II. Caracas. Editado por la Universidad Nacional Abierta, 1991. p. 39

9. Cerda H. Como Elaborar Proyectos. Diseño, Ejecución y Evaluación de Proyectos Sociales y Educativos. Bogota. Cooperativa Editorial Magisterio, 1998.

10. Orozco C, Labrador M y Palencia A. Metodología. Venezuela. Ofimax de Venezuela CA, 2002. p. 22.

11. Ander-Egg E. Introducción a las Técnicas de la Investigación Social. Buenos Aires. Humanistas, 1981.

12. Gómez C. Proyectos Factibles, Planificación, Formulación y Ejecución. Venezuela. Segunda Edición. Editorial Prográfica, 2000.p. 24.

13. Seltiz C, Jahoda M y Cook SW. Métodos de Investigación en las Relaciones Sociales. Madrid. Editorial Rialp, 1965. p.258. 
14. Seijas Z. Investigación por Muestreo. Universidad Central de Venezuela. Facultad de Ciencias Económicas y Sociales. Unidad de Reproducción y Publicación, 1998. p.90.

15. Polit D Y Hungler B. Investigación Científica en Ciencias de la Salud. México. Cuarta Edición. Editorial Interamericana. McGraw-Hill, 1994. p.198-379-646.

16. Sabino C. El Proceso de Investigación. Colombia. Segunda Edición. Editorial Panamericana, 1995. p.127.

17. Pick S y López A. Como Investigar en Ciencias Sociales. México. Quinta Edición. Editorial Trillas, 1994. p.29

18. Block A. Innovación Educativa. El sistema Integral de Enseñanza-Aprendizaje. México. Editorial Trillas, 1981.

19. Constitución de la República Bolivariana de Venezuela. Gaceta Oficial de la República de Venezuela Número 36.860. Diciembre 30, 1999.

20. Ley del Estatuto de la Función Pública. Gaceta Oficial de la República Bolivariana de Venezuela Numero 36.860. Diciembre 30, 1999.

21. Ley del Ejercicio Profesional de Enfermería. Gaceta Oficial de la República Bolivariana de Venezuela Número 38.263. Septiembre 01, 2005. 